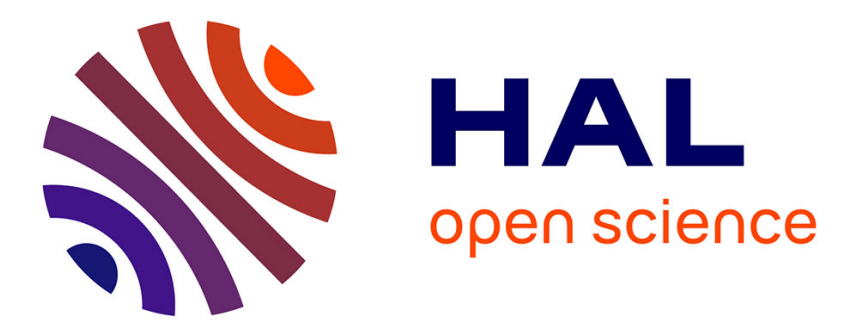

\title{
Mesure de l'énergie d'électrons dans une chambre à bulles
}

\author{
Y. Bugadov, W. Vénus, R. Arnold
}

\section{To cite this version:}

Y. Bugadov, W. Vénus, R. Arnold. Mesure de l'énergie d'électrons dans une chambre à bulles. Revue de Physique Appliquée, 1969, 4 (2), pp.302-303. 10.1051/rphysap:0196900402030201 . jpa-00243272

\section{HAL Id: jpa-00243272 https://hal.science/jpa-00243272}

Submitted on 1 Jan 1969

HAL is a multi-disciplinary open access archive for the deposit and dissemination of scientific research documents, whether they are published or not. The documents may come from teaching and research institutions in France or abroad, or from public or private research centers.
L'archive ouverte pluridisciplinaire HAL, est destinée au dépôt et à la diffusion de documents scientifiques de niveau recherche, publiés ou non, émanant des établissements d'enseignement et de recherche français ou étrangers, des laboratoires publics ou privés. 


\title{
MESURE DE L'ÉNERGIE D'ÉLECTRONS DANS UNE GHAMBRE A BULLES
}

\author{
Y. BUGADOV et W. VÉNUS, \\ C.E.R.N., Genève, \\ et R. ARNOLD, \\ C.R.N., Strasbourg.
}

Résumé. - Une calibration réalisée à l'aide de photons issus de désintégrations de pions neutres dans un volume limité de fréon a permis d'obtenir une relation entre l'énergie d'un électron et la longueur totale des traces de la gerbe engendrée par cet électron.

Abstract. - By using the photons resulting from the decay of neutral pions in a limited volume of freon, a calibration has permitted a relation to be obtained between the energy of an electron and the total length of the tracks of the shower initiated by this electron.

La somme des parcours des électrons produits dans une gerbe est en première approximation proportionnelle à l'énergie du photon primaire :

$$
E=b . L \text {. }
$$

Dans l'application de la formule (1), des difficultés existent $: i$ ) la linéarité de la formule n'est pas absolument correcte; ii) il est impossible de détecter un électron d'énergie inférieure à un certain seuil; iii) dans 
une chambre de dimensions finies, on ne peut observer qu'une partie de la gerbe produite. Le meilleur moyen de tourner ces difficultés est de mesurer des gerbes d'énergie connue et de trouver une relation empirique de la forme :

$$
E=\mathrm{f}\left(L, L_{\mathrm{p}}\right)
$$

où $L_{\mathrm{p}}$ représente l'espace disponible dans la chambre au développement de la gerbe (longueur potentielle).

Pour réaliser une calibration sous une forme donnée par (2), nous disposions d'un lot de désintégrations $\tau\left(K_{0} \rightarrow \pi^{+} \pi^{-} \pi^{0}\right)$ du méson $\mathrm{K}_{2}^{0}$, donc de photons résultant de la désintégration de pions neutres : le spectre d'énergie de ces photons s'étend de 10 à $500 \mathrm{MeV}$, leur longueur potentielle $L_{p}$ varie entre 1,5 et 9 longueurs de radiations; le liquide utilisé est le fréon $\left(X_{0}=11 \mathrm{~cm}\right)$. L'ajustement cinématique de la réaction de désintégration $\tau$ a été réalisé sans utiliser le résultat de la mesure des rayons $\gamma$ et seuls les photons dont l'incertitude sur le moment est inférieure à $15 \%$ ont été utilisés.

L'importance de la diffusion multiple rend souvent difficile la mesure de la longueur d'une trace par la méthode habituelle, c'est pourquoi nous avons mesuré les traces en projection sur la table de dépouillement au moyen d'un curvimètre; dans la valeur de $L$ utilisée, il a été tenu compte de l'angle d'enfoncement du photon.

Les critères auxquels doit satisfaire une gerbe afin de permettre la mesure sont les suivants : i) le bruit de fond doit être suffisamment faible pour ne pas fausser l'interprétation des traces courtes; ii) les gerbes issues de deux photons distincts doivent être assez nettement séparées de façon à ce que les photons secondaires n'aient pas d'origine ambiguë; iii) la gerbe ne doit pas présenter d'électron de grande énergie dont une partie seulement du parcours est visible dans la chambre; iv) la gerbe ne doit pas visiblement présenter de perte d'énergie importante par rayonnement de freinage donnant lieu à un photon non matérialisé dans la chambre; v) l'angle d'enfoncement de la gerbe doit être inférieur à $50^{\circ}$.

L'observation de la longueur des traces $L$ en fonction de l'énergie pour différents intervalles de longueur potentielle $L_{\mathrm{p}}$ nous a permis de déduire une forme simple pour l'équation (2):

$$
E=x_{1} L+\frac{x_{2}}{L_{\mathrm{p}}} L^{2}
$$

Les coefficients $x_{1}$ et $x_{2}$ ont été obtenus par ajustements de la forme (3) sur les points expérimentaux : $x_{1}=3,39 \mathrm{MeV} / \mathrm{cm}$ et $x_{2}=0,57 \mathrm{MeV} / \mathrm{cm}$. La formule (3) donne de bons résultats pour des énergies comprises entre 80 et $200 \mathrm{MeV}$, mais n'est cependant pas satisfaisante pour les autres énergies. Afin de tenir compte de l'impossibilité de détecter les électrons de faible énergie et de la variation de la probabilité pour un électron d'émettre un photon peu énergique, il convient de donner aux coefficients une variation croissante avec $E$. La forme adoptée pour les énergies de 20 à $500 \mathrm{MeV}$ est :

$$
\begin{aligned}
& E=\left[x_{1}+x_{2}\left(1-\mathrm{e}^{-\frac{L}{x_{3}}}\right)\right]\left[1+\frac{x_{4}}{L_{\mathrm{p}}} L\right] L \\
& \text { où : } \quad x_{1}=2,21 \mathrm{MeV} / \mathrm{cm} ; x_{2}=1,49 \mathrm{MeV} / \mathrm{cm} \\
& x_{3}=22 \mathrm{~cm} ; \quad x_{4}=0,19 \text {. }
\end{aligned}
$$

Les incertitudes sur la mesure sont décrites de manière satisfaisante par la relation :

$$
\Delta E=\sqrt{\left(x_{5} \cdot E\right)^{2}+\left(x_{6} \cdot E_{\mathrm{LNV}}\right)^{2}}
$$

avec les constantes $x_{5}=0,20$ et $x_{6}=0,80$ où $E_{\mathrm{LNV}}$ représente la correction apportée à l'énergie et due à la partie non visible de la gerbe :

$$
E_{\mathrm{LNV}}=E_{\mathrm{LV}} \cdot x_{4} \cdot \frac{L}{L_{\mathrm{p}}}
$$

avec : $\quad E_{\mathrm{LV}}=\left[x_{1}+x_{2}\left(1-\mathrm{e}^{-\frac{L}{x_{3}}}\right)\right] L$.

La direction de la trajectoire d'un photon dans la chambre peut être mesurée avec une très bonne précision; la distribution de la masse effective des paires de photons provenant de la désintégration d'un $\pi^{0}$ permet d'apprécier la précision de la mesure. La forme de cette distribution ( fig. 1) peut être comparée à celle d'une gaussienne d'une valeur moyenne $134 \pm 2,5 \mathrm{MeV} / \mathrm{c}^{2}$ et d'écart type $24 \mathrm{MeV} / \mathrm{c}^{2}$.

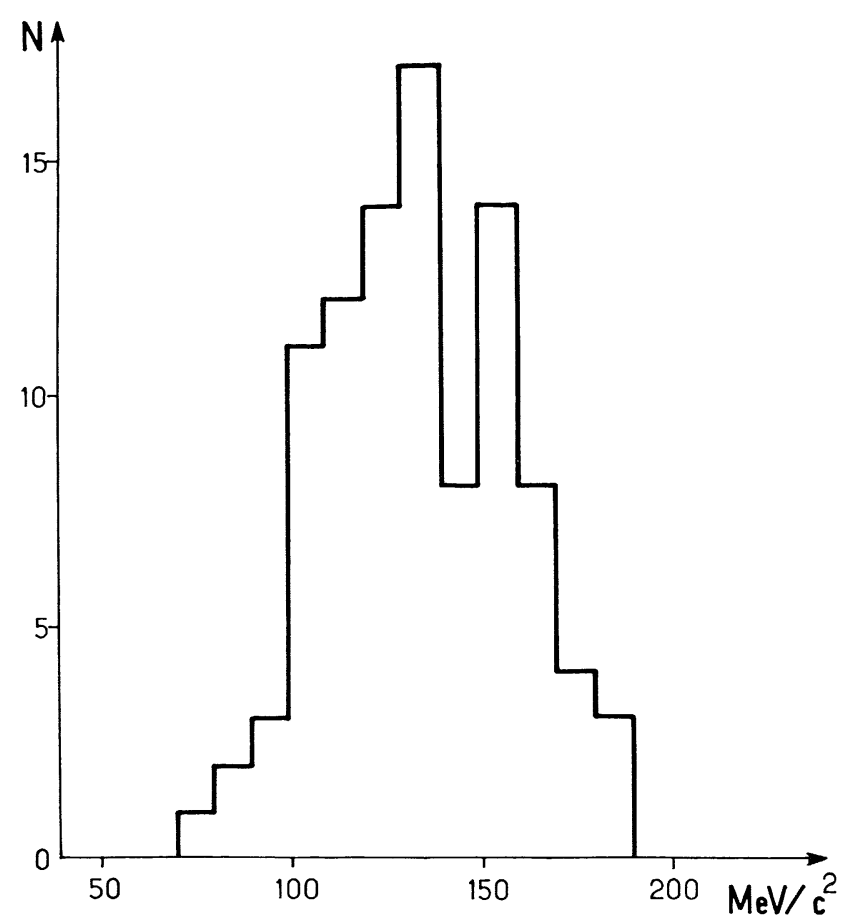

FIG. 1. - Distribution de la masse effective des paires de photons obtenue en mesurant les énergies par la méthode de la longueur totale des traces. 